\title{
Resonant tunnelling features in a suspended silicon nanowire single-hole transistor
}

Jordi Llobet, Emiljana Krali, Chen Wang, Jordi Arbiol, Mervyn E. Jones, Francesc Pérez-Murano, and Zahid A. K. Durrani

Citation: Appl. Phys. Lett. 107, 223501 (2015); doi: 10.1063/1.4936757

View online: https://doi.org/10.1063/1.4936757

View Table of Contents: http://aip.scitation.org/toc/apl/107/22

Published by the American Institute of Physics

\section{Articles you may be interested in}

Tuning piezoresistive transduction in nanomechanical resonators by geometrical asymmetries

Applied Physics Letters 107, 073104 (2015); 10.1063/1.4928709

Seebeck coefficient of one electron

Journal of Applied Physics 115, 094508 (2014); 10.1063/1.4867775

Pattern-generation and pattern-transfer for single-digit nano devices Journal of Vacuum Science \& Technology B, Nanotechnology and Microelectronics: Materials, Processing, Measurement, and Phenomena 34, 06K202 (2016); 10.1116/1.4966556

Evaluating the compressive stress generated during fabrication of Si doubly clamped nanobeams with AFM Journal of Vacuum Science \& Technology B, Nanotechnology and Microelectronics: Materials, Processing, Measurement, and Phenomena 34, 06KK02 (2016); 10.1116/1.4967930

Band parameters for III-V compound semiconductors and their alloys Journal of Applied Physics 89, 5815 (2001); 10.1063/1.1368156

High-resolution error detection in the capture process of a single-electron pump Applied Physics Letters 108, 023502 (2016); 10.1063/1.4939250

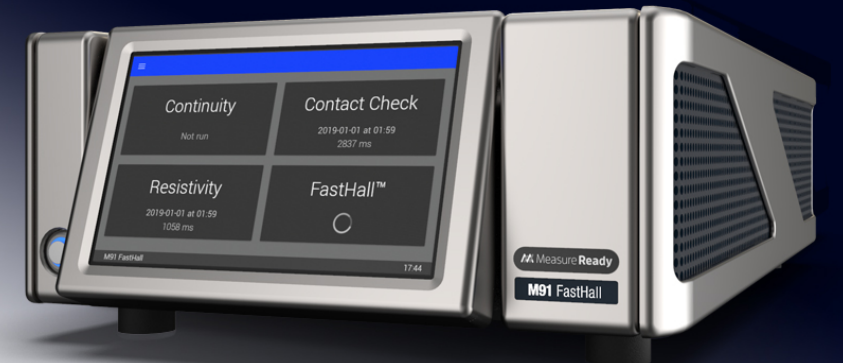

\section{Measure Ready M91 FastHall ${ }^{\mathrm{TM}}$ Controller \\ A revolutionary new instrument for complete Hall analysis}




\title{
Resonant tunnelling features in a suspended silicon nanowire single-hole transistor
}

\author{
Jordi Llobet, ${ }^{1}$ Emiljana Krali, ${ }^{2}$ Chen Wang, ${ }^{2}$ Jordi Arbiol, ${ }^{3,4}$ Mervyn E. Jones, ${ }^{2}$ \\ Francesc Pérez-Murano, ${ }^{1, a)}$ and Zahid A. K. Durrani ${ }^{2, a)}$ \\ ${ }^{1}$ Institut de Microelectrònica de Barcelona (IMB-CNM CSIC), Campus UAB, E-08193 Bellaterra, Catalonia, \\ Spain \\ ${ }^{2}$ Department of Electrical and Electronic Engineering, Imperial College London, South Kensington, \\ London SW7 2AZ, United Kingdom \\ ${ }^{3}$ Institució Catalana de Recerca i Estudis Avançats (ICREA) and Institut Català de Nanociència $i$ \\ Nanotecnologia (ICN2), Campus UAB, 08193 Bellaterra, Catalonia, Spain \\ ${ }^{4}$ CELLS-ALBA Synchrotron Light Facility, 08290 Cerdanyola, Catalonia, Spain
}

(Received 30 September 2015; accepted 14 November 2015; published online 30 November 2015)

Suspended silicon nanowires have significant potential for a broad spectrum of device applications. A suspended $p$-type Si nanowire incorporating Si nanocrystal quantum dots has been used to form a single-hole transistor. Transistor fabrication uses a novel and rapid process, based on focused gallium ion beam exposure and anisotropic wet etching, generating $<10 \mathrm{~nm}$ nanocrystals inside suspended Si nanowires. Electrical characteristics at $10 \mathrm{~K}$ show Coulomb diamonds with charging energy $\sim 27 \mathrm{meV}$, associated with a single dominant nanocrystal. Resonant tunnelling features with energy spacing $\sim 10 \mathrm{meV}$ are observed, parallel to both diamond edges. These may be associated either with excited states or hole-acoustic phonon interactions, in the nanocrystal. In the latter case, the energy spacing corresponds well with reported Raman spectroscopy results and phonon spectra calculations. (C) 2015 AIP Publishing LLC. [http://dx.doi.org/10.1063/1.4936757]

Double-clamped, suspended nanowires (NW) and nanotube structures present outstanding properties for nanomechanical resonator applications. ${ }^{1,2}$ These structures have been identified as highly promising building-blocks, for sensors with unprecedented mass detection resolution, towards the single-molecule level, ${ }^{3,4}$ for highly efficient thermoelectric "clean" energy generation, ${ }^{5,6}$ and for quantum computation. ${ }^{7,8}$ These nanostructures are also of great interest in investigations of the fundamental limits of signal processing, ${ }^{9}$ and characterising electrical, mechanical and material properties at reduced dimensions. ${ }^{10}$

Modification of suspended NW structures by the incorporation of quantum dots (QDs) provides a means to measure excited electronic states, and single-electron-phonon interactions in zero-dimensions, confined within a suspended QD cavity $^{7}$ isolated from phonon modes in the substrate. Furthermore, as these interactions are at low energies corresponding to acoustic phonon like modes, it becomes possible to probe the low-energy part of the phonon spectrum, providing a complementary technique to conventional methods, such as Raman spectroscopy. ${ }^{11}$ A range of phenomena may be investigated, e.g., phonon confinement, energy and band gaps, ${ }^{7,12}$ phonon blockade of electrical conduction, ${ }^{13}$ interactions between nanomechanical and electron motion, ${ }^{14,15}$ and decoherence effects in quantum computation devices. ${ }^{7}$ While suspended QDs have been widely investigated in materials such as carbon nanotubes $(\mathrm{CNTs}),{ }^{16}$ there are comparatively few investigations in $\mathrm{Si}^{7}{ }^{71-19}$ Previous work has concentrated on $n$-type Si material only, using lithographically patterned QDs in crystalline $\mathrm{Si}^{7,}, 17,18$ "naturally" formed QDs in nanocrystalline $\mathrm{Si}^{17}$ and

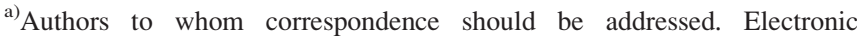
addresses: francesc.perez@csic.es and z.durrani@imperial.ac.uk
}

QDs formed by disorder. ${ }^{19}$ However, suspended $p$-type single hole transistors have not been investigated previously and even for the case of non-suspended devices, ${ }^{20-22}$ there are relatively few investigations compared with $n$-type SETs. ${ }^{23,24}$ Single-hole transistors extend the single-electron and QD family of devices to include complementary $n$ - and $p$-type devices, greatly enhancing flexibility in the design of few-electron, nanoelectronic systems. ${ }^{24,25}$ In addition, fundamental differences exist in the physical properties of $\mathrm{Si}$ single-hole transistors in comparison with single-electron transistors, associated with a different effective mass for holes, and the requirement for valence band potential barriers. ${ }^{20} \mathrm{In} \mathrm{Si}$, for a QD of radius $r$, the reduced hole effective mass implies increased excited state energy $E \sim\left(\pi^{2} \hbar^{2}\right) /\left(2 m r^{2}\right)$, improving the likelihood of observing resonant tunnelling effects through the excited states. Furthermore, in the case of Si nanocrystal single-hole transistors, information can be extracted on the nature of the nanocrystal surface, as either the presence of hole traps or a $\mathrm{SiO}_{2}$ layer on the surface is essential to create valence band tunnel barriers.

This paper presents the electrical characteristics of a suspended Si NW single-hole transistor incorporating $<10 \mathrm{~nm}$ QDs. Coulomb diamonds ${ }^{24,25}$ with energy $\sim 27 \mathrm{meV}$ and resonant tunnelling features with energy $\sim 10 \mathrm{meV}$, are observed. Resonant tunnelling features form conductance lines parallel to both diamond edges, not previously observed in suspended Si QDs. These may be associated with either excited hole states or hole-acoustic phonon interactions, within a single dominant nanocrystal QD. For the excited state model, states both above and below the Coulomb gap are needed to create lines parallel to both diamond edges. For the hole-phonon interaction model, both phonon emission and absorption may occur to create lines parallel to both diamond edges. This provides an additional mechanism to 

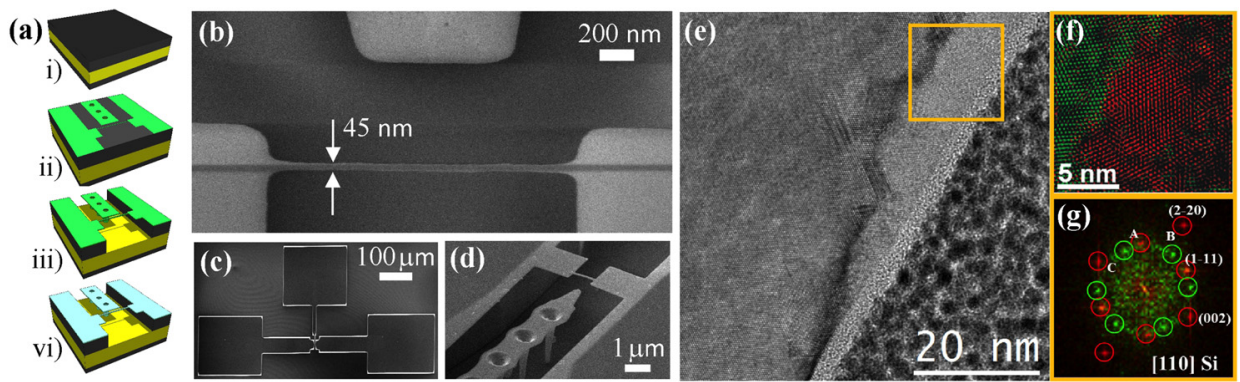

FIG. 1. (a) Schematic illustration of four steps during device fabrication process sequence, SEM micrographs showing (b) close-up of suspended Si NW and gate, (c) overall device, and (d) suspended gate showing pillar supports. (e) HRTEM image obtained at the area of one of the nanowires. After recrystallization, the NW presents stacking faults and twin boundaries as well as polycrystalline domains. The area selected in orange and zoomed on the right side shows a perfect [110] oriented grain (green), in contact with an off-axis silicon grain (partially rotated). (f) Corresponds to a combined reconstructed real space image obtained from the FFT spots (green or red) selected in the combined power spectra of $(\mathrm{g})$. (g) The combined power spectra obtained on the orange selected area of the HRTEM image. Both the [110] oriented (green spots) and the off-axis domains (red spots) can be visualized.

create both sets of lines, increasing the likelihood of their occurrence. For the hole-phonon interaction case, a lowenergy, acoustic phonon-like mode $\hbar \omega_{p} \sim 10 \mathrm{meV}$ can be associated with the dominant nanocrystal in the device. This corresponds well with reported Raman spectroscopy results ${ }^{11}$ and phonon spectra calculations in Si nanocrystals. ${ }^{26,27}$

The fabrication sequence and device structure is shown in Fig. 1(a), based on a novel focused ion beam (FIB) exposure and wet chemical etching process. ${ }^{28}$ This provides a fast prototyping method for nanomechanical devices and for generating nanocrystals within suspended Si NWs. The starting substrates were taken from (110) SOI wafers, with top Si thickness $2 \pm 0.5 \mu \mathrm{m}$ and nominal resistivity $9.5 \times 10^{-4}-11 \times 10^{-4} \Omega \mathrm{m}$ ( $p$-type), and buried $\mathrm{SiO}_{2}$ thickness $2 \mu \mathrm{m} \pm 5 \%$. The surface was patterned by FIB at a low exposure dose, creating amorphous Si areas with high gallium concentration. Exposed areas were resistant to wet Si etching and formed the device structural parts. Following exposure, suspended Si NWs were defined using wet anisotropic etching. With the appropriate device orientation, the nanowire remained completely suspended, while the clamping sites and pads were fully defined across the top Si layer. The suspended NW thickness was dictated by the range of implanted gallium, $\sim 40 \mathrm{~nm}$. Finally, annealing and boron doping of the device at $850{ }^{\circ} \mathrm{C}-1000^{\circ} \mathrm{C}$ recovered crystallinity and electrical conductivity, with final NW resistivity $\sim 4 \times 10^{-3} \Omega \mathrm{m}$.

A SEM image of the suspended Si NW measured in this work is shown in Fig. 1(b). The NW was $2.15 \mu \mathrm{m} \times 45 \mathrm{~nm}$ $\times 40 \mathrm{~nm}$ in size, and suspended $1.8 \mu \mathrm{m}$ above the $\mathrm{SiO}_{2}$ substrate. A lower magnification SEM image of a similar device (Fig. 1(c)) also shows pads and contact lines. A gate electrode was defined in close proximity $(700 \mathrm{~nm})$ to the NW. In order to avoid its collapse, sustaining posts were defined by a combination of FIB implantation and milling (Fig. 1(d)).

The suspended NW structure was nanocrystalline due to amorphisation from the gallium implantation and subsequent recrystallisation. Figure 1(e) shows a high-resolution transmission electron microscopy (HRTEM) micrograph of the exposed and annealed area after recrystallisation. The NW volume presents several crystal defects, such as stacking faults and twin boundaries as reported previously, ${ }^{28}$ with additionally, polycrystalline domains observed (see Fig. 1(e) right). Here, the bright, diagonal region corresponds to the recrystallised area, $\sim 60 \mathrm{~nm}$ length and $\sim 10 \mathrm{~nm}$ in maximum width. This region is composed of smaller nanocrystals randomly oriented, with sizes ranging from $\sim 5$ to $10 \mathrm{~nm}$. In the region marked by the square, an oriented crystal along the [110] zone axis faces another off-axis crystallite. The misoriented crystallite shows the characteristic HRTEM features of a $\mathrm{Si}$ bicrystalline nanoparticle rotated $60^{\circ}$ from the [110] zone axis. ${ }^{29}$ Bicrystalline Si nanodomains are common nanoparticles, and nanowires consist of nanostructures divided into two identical mirror halves joined by a $\{111\}$ twin boundary. ${ }^{29-32}$ In our previous work, Fig. 3(d) of Ref. 28, a brighter region showing several Moirée fringes is reported, corresponding to different crystals overlapping at random orientations. In this case, the size of the crystals also lies in the range between $\sim 5$ and $10 \mathrm{~nm}$.

Electrical characterisation was performed using a CTICryogenics closed cycle helium cryostat and an Agilent 4155B parameter analyser. "Coulomb diamond" single-hole tunnelling characteristics in a $40 \mathrm{~nm} \times 45 \mathrm{~nm} \times 2.15 \mu \mathrm{m}$ suspended NW device at $10 \mathrm{~K}$ are shown in Figs. 2(a)-2(d). Similar behaviour is also observed in a second device. The drain-source current $\left(I_{d s}\right)$ vs. drain-source $\left(V_{d s}\right)$ and side-gate voltage $\left(V_{g s}\right)$ are shown in Fig. 2(a), and the drain-source conductance $\left(g_{d s}\right)$ vs. $V_{d s}, V_{g s}$ in Figs. 2(b)-2(d). The $I_{d s}-V_{d s}$, $V_{g s}$ characteristics (Fig. 2(a)) show non-linear $I_{d s}-V_{d s}$ curves, modulated periodically as $V_{g s}$ varies from 0 to $20 \mathrm{~V}$. The $I_{d s}-V_{d s}$ curves are broadly symmetric around $V_{d s}=0 \mathrm{~V} . I_{d s}$ oscillates with $V_{g s}$ with a large period $\Delta V_{g s l} \sim 7 \mathrm{~V}$, and a finer, superimposed period $\Delta V_{g s l} \sim 1.1 \mathrm{~V}$. The $g_{d s}$ vs. $V_{d s}$, $V_{g s}$ characteristics (Figs. 2(b)-2(d)) are shown using a threedimensional plot (b) and using colour scale plots (c) and (d) to highlight trapezoidal Coulomb diamond regions and associated features. Three Coulomb diamonds are observed, where diamonds 1 and 2 (marked by white solid lines) are similar, and the diamond centred at $V_{g s}=5 \mathrm{~V}$ is reduced in width along $V_{d s}$. The fine oscillation in $I_{d s}$ with $V_{g s}$ (Fig. 2(a)) also occurs in $g_{d s}$, leading to an oscillation of the diamond edges in Figs. 2(c) and 2(d). Fine structure is also observed in Figs. 2(b) and 2(c), forming lines parallel to and outside the diamond edges, which will be discussed later.

The $g_{d s}-V_{g s}$ characteristics at selected values of $V_{d s}$ are shown in Figs. 3(a) and 3(b). Fine structure peaks are observed even within the diamond regions, e.g., at $V_{g s} \approx 14 \mathrm{~V}$ (Fig. 3(b)). The $g_{d s}-V_{d s}$ characteristics at $V_{g s}=0 \mathrm{~V}$ from 10 to $80 \mathrm{~K}$ are shown in Fig. 3(c). It is seen that both the central Coulomb blockade valley and fine structure persist to $\sim 40 \mathrm{~K}$. 
(a)

(c)
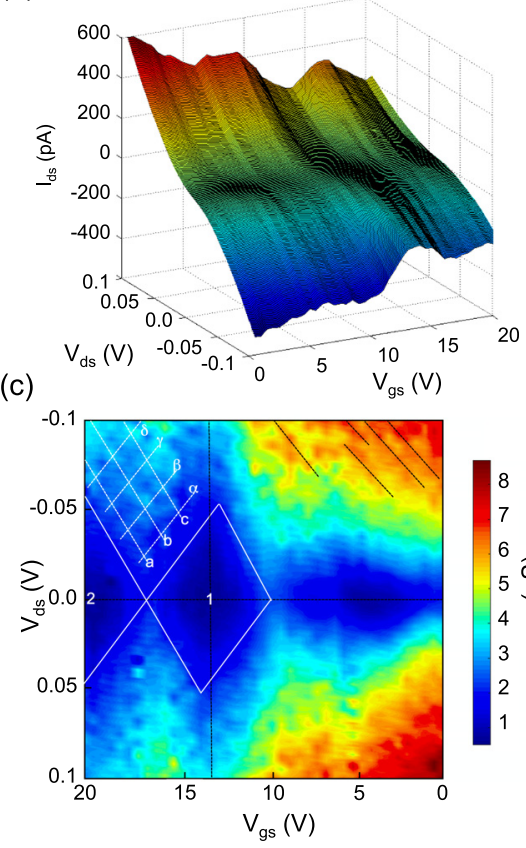

(b)

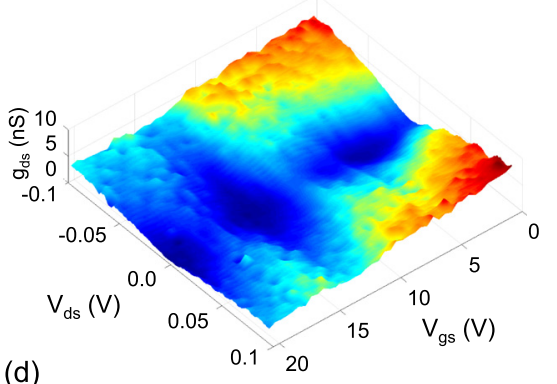

(d)

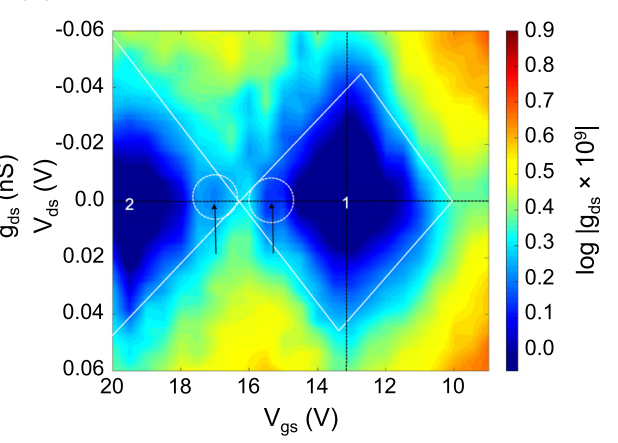

FIG. 2. Single-hole charging in the gated Si NW at $10 \mathrm{~K}$. (a) $I_{d s}$ vs. $V_{d s}$ and $V_{g s}$ characteristics, (b) $g_{d s}$ vs. $V_{d s}$ and $V_{g s}$ characteristics, and (c) $g_{d s}$ vs. $V_{d s}$ and $V_{g s}$ characteristics on a colour scale. Coulomb diamonds (labeled 1 and 2) are indicated by solid white lines and resonant tunnelling features are indicated using dashed white $(a-c$ and $\alpha-\delta$ ) and dashed black lines (in top right quadrant). These features can also be seen in (b). (d) Contour plot of $\log \left|g_{d s}\right|$ vs. $V_{d s}$ and $V_{g s}$ characteristics in the region of Coulomb diamonds 1 and 2 in (c).
The device characteristics can be associated with singlehole charging of a dominant island within the NW. Coulomb diamonds 1 and 2 are similar, implying a single, dominant island. The additional diamond centred at $V_{g s}=5 \mathrm{~V}$ is slightly wider along $V_{g s}$ and narrower along $V_{d s}$, implying some change in this case for the island gate $\left(C_{g}\right)$ and tunnel $\left(C_{1}\right.$ and $\left.C_{2}\right)$ capacitances with $V_{g s}{ }^{24,25} C_{1}, C_{2}$, and $C_{g}$ are now extracted ${ }^{25}$ from diamonds 1 and 2 . The diamond edges (solid white lines) are drawn such that they pass through the mid-point between the diamonds and are parallel to $g_{d s}$ contours. The diamond 1 width is $\Delta V_{g s l}=6.9 \mathrm{~V}$, implying $C_{g}=e / \Delta V_{g s l}=0.023 \mathrm{aF}$. The total island capacitance $C_{t}=C_{1}+C_{2}+C_{g}$ can be found using the $V_{d s}$ value at the top or bottom corner, $V_{d s l}=e / C_{t}=0.053 \mathrm{~V}$, giving $C_{t}=3 \mathrm{aF}$. The $V_{d s}$ value of the intersection between the diamond edge and the vertical line through the diamond centre, $V_{d s i}$ $=e /\left(2 C_{2}+C_{g}\right)=0.043 \mathrm{~V}$, gives $C_{2}=1.85 \mathrm{aF}$. Hence, using $C_{t}=3 \mathrm{aF}$, we find $C_{2}=1.13 \mathrm{aF}$. Finally, island single hole charging energy is $E_{c}=e^{2} / 2 C_{t}=27 \mathrm{meV}$.

The origin of the QD creating the characteristics of Fig. 2 is now discussed. Holes from nanocrystals can be trapped at grain boundaries (GBs) in the nanocrystalline Si NW device channel, creating potential barriers. ${ }^{33,34}$ Many nanocrystals with a distribution of barrier heights exits along the NW (Fig. 3(d-i)), e.g., nanocrystal "A" has higher barriers than "B." Conduction occurs via percolation through nanocrystals with the lowest GB barriers. ${ }^{35}$ As the gate bias is increased, the valence band edge energy decreases, with accompanying depletion of hole concentration within the nanocrystals and an increase in the GB barrier seen by the holes (Fig. 3(d-ii)). ${ }^{35}$ This picture is supported by the trend to reduced current with increasing $V_{g s}$ (Fig. 2(a)). The electrical characteristics of Fig. 2 occur at the point where single-hole charging has only just begun, and only one nanocrystal ("A" in Fig. 3(d-ii)) is sufficiently isolated. At higher $V_{g s}$ values, additional QDs would become isolated and a multiple tunnel junction (MTJ) would form, leading to more complex Coulomb diamond patterns. ${ }^{36}$

The extracted capacitances may be used to estimate QD size and hence correlated with the nanocrystalline structure.
The gate capacitance for the entire NW, calculated using a finite-element capacitance calculation, $C_{N W}=10 \mathrm{aF}$, implies an NW capacitance per unit length $c_{N W} \sim C_{N W} / l_{N W} \sim 4.7 \mathrm{aF} / \mu \mathrm{m}$. As $C_{g}$ for the charging island is only $0.023 \mathrm{aF}$, this implies a very small island length $l_{i} \sim 5 \mathrm{~nm}$, similar to the nanocrystal grain size. ${ }^{28}$ Furthermore, assuming a spherical island of diameter $d_{i}$ with self-capacitance $C_{i}=C_{t}=2 \pi \varepsilon_{0} \varepsilon_{r} d_{i}=3 \mathrm{aF}$ gives $d_{i} \sim 4.5 \mathrm{~nm} \sim l_{i}$. The charging island can therefore be associated with a Si nanocrystal within the NW.

Features for negative $V_{d s}$ are indicated by dashed lines in Fig. 2(c). Here, lines lying between diamonds 1 and 2 (labelled $\alpha-\delta$ and $a-c$ ) form a subsidiary diamond-like pattern. Similar, although less clear, features can be observed for positive $V_{d s}$, e.g., in the range $0.02 \mathrm{~V}<V_{d s}<0.1 \mathrm{~V}$ and $15 \mathrm{~V}<V_{g s}<20 \mathrm{~V}$. In principle, the fine structure may be expected to occur symmetrically in $V_{d s}$. However, as the structure is superimposed on a background variation in $g_{d s}$ created by the Coulomb diamonds, the background and any asymmetry in this influences the clarity of the structure. A larger change in background $g_{d s}$ for positive $V_{d s}$, in the region between Coulomb diamonds 1 and 2 (Figs. 2(b) and 2(c)), also seen in Figs. 3(a) and 3(b), reduces clarity of the fine structure.

Typically, fine structure of the form of Fig. 2(c) is caused by electron tunnelling through excited states in the underlying QD, or phonon assisted inelastic electron tunnelling through the QD. ${ }^{37-39}$ We note that the pattern formed by the lines $\alpha-\delta$ and $a-c$ very closely resembles phonon assisted tunnelling patterns in suspended CNT QDs. ${ }^{38}$ Additional features are observed within the Coulomb diamonds. Near the central point between diamonds 1 and 2 (Fig. 2(d)), circular features (arrowed, and marked by dashed circles) are formed due to conductance lines cutting into the diamonds. Regions of higher conductance also extend past the nominal diamond edge (white line), e.g., along the top left edge of diamond 1.

The fine structure in Figs. 2 and 3 can be created by excited states, or phonon assisted inelastic electron tunnelling in the QD. Conductance lines parallel to Coulomb diamond edges ${ }^{37}$ are associated mainly with additional resonant 
(a)

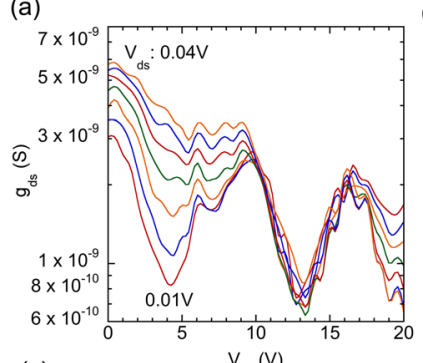

(c)

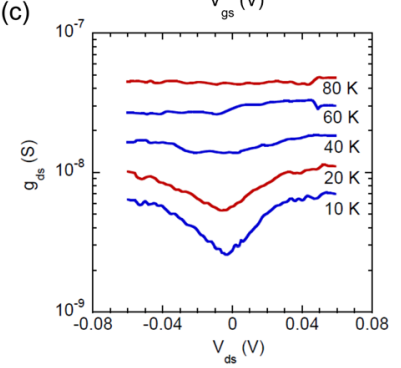

(b)

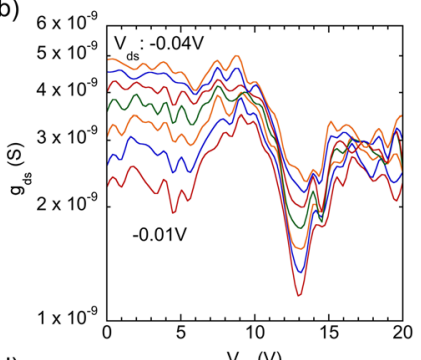

(d)

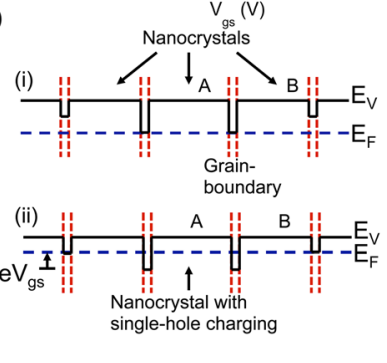

FIG. 3. Drain source conductance characteristics (a) $g_{d s}$ vs. $V_{g s}$ at selected values of $V_{d s}=0.01 \mathrm{~V}-0.04 \mathrm{~V}$, in increments of $0.005 \mathrm{~V}$, (b) $g_{d s} \mathrm{vs}$. $V_{g s}$ at selected values of $V_{d s}=-0.01 \mathrm{~V}$ to $-0.04 \mathrm{~V}$ in increments of $-0.005 \mathrm{~V}$, (c) $g_{d s}$ vs. $V_{d s}$ characteristics from 10 to $80 \mathrm{~K}$, at $V_{g s}=0 \mathrm{~V}$. (d) Distribution of grain boundary potential barriers, shown schematically, (i) at zero gate bias and (ii) with applied gate bias $V_{g s}>0 \mathrm{~V}$. As $V_{g s}$ increases, single-hole charging occurs first in the nanocrystal (marked A) with the highest potential barriers.

tunnelling paths through excited states. While in many cases, asymmetrical QD tunnel barriers cause lines parallel to only one diamond edge, ${ }^{37}$ less frequently, the presence of symmetrical barriers creates lines parallel to both edges ${ }^{40}$ in a manner similar to our data. Alternatively, in suspended QDs in $\mathrm{CNTs}^{38}{ }^{38}$ phonon assisted tunnelling can also create roughly equidistant conductance lines parallel to both diamond edges, very similar to our results. In CNTs, the energy scale of the lines enables differentiation between excited states and phonon-assisted tunnelling. ${ }^{38}$ At elevated temperatures, phonon absorption causes conductance lines to extend into the Coulomb diamonds. ${ }^{38}$ Finally, while fine structure has been reported in suspended QDs in $n$-type $\mathrm{Si}^{17-19}$ lines parallel to the Coulomb diamonds have not been reported.

Energy band diagrams for the single-hole transistor Coulomb diamond are shown schematically in Fig. 4. A valence-band electron picture is shown for simplicity, with carrier transport from source to drain. Hole transport would occur concurrently along the same path, but from drain to source. $E_{N}$ and $E_{N+1}$ are the island chemical potentials for $N$ and $N+1$ electrons, respectively. $E_{F S}$ and $E_{F D}$ are the source and drain Fermi energies. Dotted/solid lines lying above and below $E_{N+1}$ and $E_{N}$ show normally empty/filled states in an excited states picture. ${ }^{37,39}$ In the phonon assisted tunnelling picture ${ }^{37}$ these correspond to a ladder of phonon absorption (dotted lines) and emission (solid lines) levels, $E_{p n}=n \hbar \omega_{p}$ where $\omega_{p}$ is the fundamental phonon mode. Other absorption/emission levels can also exist, lying within $E_{c}$ (not shown for clarity). At positions (a) and (c), Coulomb blockade is overcome, with $N+1$ and $N$ electrons on the island, respectively. Position (b) corresponds to Coulomb blockade imposed fully. Position (e) shows the condition along the top left diamond edge, such that $E_{N+1}$ remains aligned with $E_{F S}$. Along the top right edge, $E_{N}$ remains aligned with $E_{F D}$

(d)

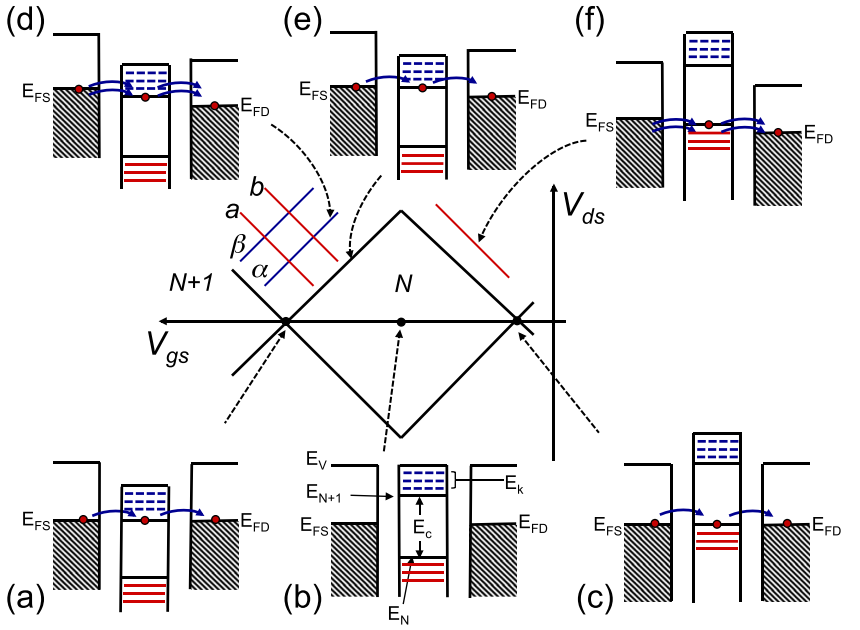

FIG. 4. Energy band diagrams for the single-hole transistor at specific points within a Coulomb diamond, with pathways for tunnelling shown by arrows. (a) $V_{d s}=0 \mathrm{~V}, N+1$ valence band electrons on the island, and Coulomb blockade overcome. (b) Coulomb blockade fully imposed. (c) $V_{d s}=0 \mathrm{~V}, N$ valence band electrons on the island, and Coulomb blockade overcome. (d) Resonant tunnelling features parallel to the top left diamond edge. (e) Along the top left diamond edge. (f) Resonant tunnelling features parallel to the top right diamond edge.

(position (f)). In Fig. 2(c), for lines $\alpha-\delta$ parallel to the top left edge, tunnelling occurs through excited states above the Coulomb gap and within the $E_{F S}-E_{F D}$ window, with $N$ electrons on the QD. For lines $a-c$ parallel to the top right edge, tunnelling occurs through excited states below the Coulomb gap and within the $E_{F S}-E_{F D}$ window, with $N+1$ electrons on the QD.

The energy spacing between adjacent lines is now extracted. By extending the lines to find the intersection points with the adjacent diamond edge, the $V_{d s}$ value of this point gives the energy of the state relative to the Coulomb gap. ${ }^{39}$ The energies for the first lines ( $a$ and $\alpha$ ) are $E_{a}=10 \mathrm{meV}$ and $E_{\alpha}=15 \mathrm{meV}$. The average energy spacing is $\Delta_{l}=11.2 \mathrm{meV}$ and $\Delta_{2}=15 \mathrm{meV}$, in groups $a-c$ and $\alpha-\delta$, respectively.

These energy scales are considered first within the excited state model. Here, a spherical QD of radius $r$ may be assumed to represent the charging nanocrystal in our device. The energy of the first line $E_{a}$ may then be associated with the first confinement state energy $E_{l}=\left(\pi^{2} \hbar^{2}\right) /\left(2 m r^{2}\right)$ in a spherical QD, giving $r \sim 7-8 \mathrm{~nm}$ (using an effective mass for holes of 0.54). This is similar to the nanocrystal size and to the value extracted using $E_{c}$. On the other hand, in the phonon-assisted tunnelling model, $E_{p l}=\hbar \omega_{p}=2 \pi \hbar f_{p}=E_{a}$ and $E_{\alpha}$, giving a phonon fundamental frequency $f_{p}=2.4 \mathrm{THz}$ and $3.6 \mathrm{THz}$, respectively. Furthermore, some evidence exists for lines extending into the diamonds past their edges (Fig. 2(d)), a signature of phonon-assisted tunnelling. ${ }^{38}$ Additional lines also cut into the diamonds, defining the arrowed features in Fig. 2(d).

A phonon assisted tunnelling model is also supported by comparing extracted energy scales to experimental and theoretical reports on the phonon spectrum of $\mathrm{Si}$ nanocrystals. ${ }^{11,26,27}$ Raman measurements have shown acoustic phonon peaks at $2.5-3.7 \mathrm{meV}$, in Si nanocrystals $\sim 3-5 \mathrm{~nm}$ in diameter. ${ }^{11}$ Theoretical calculations of the phonon density of states (DOS) in $\sim 2-3 \mathrm{~nm}$ Si nanocrystals predict low energy acoustic-phonon peaks at $6-12 \mathrm{meV}^{26,27}$ For both Raman 
measurements ${ }^{11}$ and theoretical calculations, ${ }^{26,27}$ the first phonon peak energy is similar to our extracted value, $E_{p l}$ $=10-15 \mathrm{meV}$, supporting a phonon-assisted tunnelling model.

In conclusion, a suspended $p$-type $\mathrm{Si}$ NW single-hole transistor, which includes $<10 \mathrm{~nm}$ nanocrystal QDs, was fabricated using focused gallium ion beam exposure and anisotropic wet etching. Electrical characteristics at $10 \mathrm{~K}$ showed Coulomb diamonds having a charging energy $\sim 27 \mathrm{meV}$, associated with a single dominant nanocrystal. Resonant tunnelling features with energy spacing $\sim 10 \mathrm{meV}$ were observed parallel to both diamond edges. While the patterns formed by these features may be associated with excited electron states, they may also be associated with hole-acoustic phonon interactions in the nanocrystal. In the latter case, the patterns are very similar to results observed in suspended CNT QDs, and the energy scale corresponds well to reported Raman spectroscopy results and phonon spectra calculations.

The research leading to these results received funding from the European Union's Seventh Framework Programme FP7/2007-2013, under Grant Agreement No. 318804 (SNM).

${ }^{1}$ A. Husain, J. Hone, H. W. C. Postma, X. M. H. Huang, T. Drake, M. Barbic, A. Scherer, and M. L. Roukes, "Nanowire-based very-high-frequency electromechanical resonator," Appl. Phys. Lett. 83, 1240-1242 (2003).

${ }^{2}$ V. Sazonova, Y. Yaish, H. Üstünel, D. Roundy, T. A. Arias, and P. L. McEuen, "A tunable carbon nanotube electromechanical oscillator," Nature 431, 284-287 (2004).

${ }^{3}$ J. Chaste, A. Eichler, J. Moser, G. Ceballos, R. Rurali, and A. Bachtold, "A nanomechanical mass sensor with yoctogram resolution," Nat. Nanotechnol. 7, 301-304 (2012).

${ }^{4}$ M. S. Hanay, S. Kelber, A. K. Naik, D. Chi, S. Hentz, E. C. Bullard, E. Colinet, L. Duraffourg, and M. L. Roukes, "Single-protein nanomechanical mass spectrometry in real time," Nat. Nanotechnol. 7, 602-608 (2012).

${ }^{5}$ A. I. Hochbaum, R. Chen, R. D. Delgado, W. Liang, E. C. Garnett, M. Najarian, A. Majumdar, and P. Yang, "Enhanced thermoelectric performance of rough silicon nanowires," Nature 451, 163-167 (2008).

${ }^{6}$ E. Krali and Z. A. K. Durrani, "Seebeck coefficient in silicon nanowire arrays," Appl. Phys. Lett. 102, 143102 (2013).

${ }^{7}$ J. Ogi, T. Ferrus, T. Kodera, Y. Tsuchiya, K. Uchida, D. A. Williams, S. Oda, and H. Mizuta, "Experimental observation of enhanced electronphonon interaction in suspended Si double quantum dots," Jpn. J. Appl. Phys. 49, 045203 (2010).

${ }^{8}$ Y. Y. Liao, Y. N. Chen, W. C. Chou, and D. S. Chuu, "Decoherence of a charge qubit embedded inside a suspended phonon cavity," Phys. Rev. B 77, 033303 (2008).

${ }^{9}$ V. Gouttenoire, T. Barois, S. Perisanu, J. L. Leclercq, S. T. Purcell, P. Vincent, and A. Ayari, "Digital and FM demodulation of a doubly clamped single-walled carbon-nanotube oscillator: Towards a nanotube cell phone," Small 6, 1060-1065 (2010).

${ }^{10}$ M. Sansa, M. Fernández-Regúlez, J. Llobet, Á. San Paulo, and F. PérezMurano, "High-sensitivity linear piezoresistive transduction for nanomechanical beam resonators," Nat. Commun. 5, 4313 (2014).

${ }^{11}$ M. Fujii, Y. Kanzawa, S. Hayashi, and K. Yamamoto, "Raman scattering from acoustic phonons confined in Si nanocrystals," Phys. Rev. B 54, R8373 (1996)

${ }^{12}$ D. M. Sagar, J. M. Atkin, P. K. B. Palomaki, N. R. Neale, J. L. Blackburn, J. C. Johnson, A. J. Nozik, M. B. Raschke, and M. C. Beard, "Quantum confined electron-phonon interaction in silicon nanocrystals," Nano Lett. 15, 1511-1516 (2015).

${ }^{13}$ S. Uno, N. Mori, K. Nakazato, N. Koshida, and H. Mizuta, "Reduction of acoustic-phonon deformation potential in one-dimensional array of $\mathrm{Si}$ quantum dot interconnected with tunnel oxides," J. Appl. Phys. 97, 113506 (2005).

${ }^{14}$ E. M. Weig, R. H. Blick, T. Brandes, J. Kirschbaum, W. Wegscheider, M. Bichler, and J. P. Kotthaus, "Single-electron-phonon interaction in a suspended quantum dot phonon cavity,” Phys. Rev. Lett. 92, 046804 (2004).
${ }^{15}$ A. D. Armour, M. P. Blencowe, and Y. Zhang, "Classical dynamics of a nanomechanical resonator coupled to a single-electron transistor," Phys. Rev. B 69, 125313 (2004)

${ }^{16}$ A. K. Huttel, M. Poot, B. Witkamp, and H. S. J. van der Zant, "Nanoelectromechanics of suspended carbon nanotubes," New J. Phys. 10, 095003 (2008).

${ }^{17}$ J. Ogi, Y. Tsuchiya, S. Oda, and H. Mizuta, "Single-electron tunnelling via quantum dot cavities built on a silicon suspension nanobridge," Microelectron. Eng. 85, 1410-1412 (2008).

${ }^{18}$ J. Ogi, M. A. Ghiass, Y. Tsuchiya, K. Uchida, S. Oda, and H. Mizuta, "Suspended quantum dot fabrication on a heavily-doped silicon nanowire by suppressing unintentional quantum dot formation,” Jpn. J. Appl. Phys. 49, 044001 (2010).

${ }^{19}$ A. Tilke, L. Pescini, A. Erbe, H. Lorenz, and R. H. Blick, "Electron-phonon interaction in suspended highly doped silicon nanowires," Nanotechnology 13, 491-494 (2002).

${ }^{20} \mathrm{H}$. Ishikuro and T. Hiramoto, "On the origin of tunneling barriers in silicon single electron and single hole transistors," Appl. Phys. Lett. 74, 1126-1128 (1999).

${ }^{21}$ H. Harata, M. Saitoh, and T. Hiramoto, "Silicon single-hole transistor with large coulomb blockade oscillations and high voltage gain at room temperature,” Jpn. J. Appl. Phys. 44, L640-L642 (2005).

${ }^{22}$ F. A. Zwanenburg, A. A. van Loon, G. A. Steele, C. E. W. M. van Rijmenam, T. Balder, Y. Fang, C. M. Lieber, and L. P. Kouwenhoven, J. Appl. Phys. 105, 124314 (2009).

${ }^{23}$ Z. Zhong, Y. Fang, W. Lu, and C. M. Lieber, Nano Lett. 5, 1143-1146 (2005).

${ }^{24}$ Y. Takahashi, Y. Ono, A. Fujiwara, and H. Inokawa, J. Phys.: Condens. Matter 14, R995-R1033 (2002).

${ }^{25}$ Z. A. K. Durrani, Single-Electron Devices and Circuits in Silicon (Imperial College Press, London, 2010).

${ }^{26}$ A. Valentin, J. Sée, S. Galdin-Retailleau, and P. Dollfus, "Study of phonon modes in silicon nanocrystals using the adiabatic bond charge model," J. Phys.: Condens. Matter 20, 145213 (2008).

${ }^{27}$ A. A. Prokofiev, A. N. Poddubny, and I. N. Yassievich, "Phonon decay in silicon nanocrystals: Fast phonon recycling," Phys. Rev. B 89, 125409 (2014).

${ }^{28}$ J. Llobet, M. Sansa, M. Gerbolés, N. Mestres, J. Arbiol, X. Borrisé, and F. Pérez-Murano, Nanotechnology 25, 135302 (2014).

${ }^{29}$ H. Kohno, N. Ozaki, H. Yoshida, K. Tanaka, and S. Takeda, Cryst. Res. Technol. 38, 1082 (2003).

${ }^{30}$ A. H. Carim, K. K. Lew, and J. M. Redwing, "Bicrystalline silicon nanowires," Adv. Mater. 13, 1489 (2001).

${ }^{31}$ S. Conesa-Boj, I. Zardo, S. Estrade, L. Wei, P. J. Alet, P. R. I. Cabarrocas, J. R. Morante, F. Peiro, A. F. I. Morral, and J. Arbiol, "Defect formation in Ga-catalyzed silicon nanowires," Cryst. Growth Des. 10, 1534-1543 (2010).

${ }^{32}$ M. Y. Bashouti, C. A. Garzuzi, M. de la Mata, J. Arbiol, J. Ristein, H. Haick, and S. Christiansen, "The role of silicon nanowire diameter for alkyl (chain lengths: C1-C18) passivation efficiency through Si-C bonds," Langmuir 31, 2430 (2015).

${ }^{33}$ Y. T. Tan, T. Kamiya, Z. A. K. Durrani, and H. Ahmed, "Room temperature nanocrystalline silicon single-electron transistors," J. Appl. Phys. 94, 633-637 (2003).

${ }^{34}$ M. A. H. Khalafalla, H. Mizuta, and Z. A. K. Durrani, "Identifying singleelectron charging islands in a two-dimensional network of nanocrystalline silicon grains using Coulomb oscillation fingerprints," Phys. Rev. B 74, 35316 (2006).

${ }^{35}$ Z. A. K. Durrani and M. A. Rafiq, "Electronic transport in silicon nanocrystals and nanochains," Microelectron. Eng. 86, 456-466 (2009).

${ }^{36}$ M. B. A. Jalil, H. Ahmed, and M. J. Wagner, "Analysis of multiple-tunnel junctions and their application to bidirectional electron pumps," Appl. Phys. 84, 4617-4624 (1998).

${ }^{37}$ C. C. Escott, F. A. Zwanenburg, and A. Morello, "Resonant tunnelling features in quantum dots," Nanotechnology 21, 274018 (2010).

${ }^{38}$ R. Leturcq, C. Stampfer, K. Inderbitzin, L. Durrer, C. Hierold, E. Mariani, M. G. Schultz, F. von Oppen, and K. Ensslin, "Franck-Condon blockade in suspended carbon nanotube quantum dots," Nat. Phys. 5, 327-331 (2009).

${ }^{39}$ L. E. P. Kouwenhoven, C. M. Marcus, P. L. McEuen, S. Tarucha, and M. Robert, Electron Transport in Quantum Dots (Mesoscopic Electron Transport, Kluwer, 1997).

${ }^{40}$ L. E. P. Kouwenhoven, D. G. Austing, and S. Tarucha, "Few-electron quantum dots," Rep. Prog. Phys. 64, 701-736 (2001). 